Research Article

\title{
Lipolytic and Lipophagic Effects of Pinellia ternata Pharmacopuncture on Localized Adiposity
}

\author{
Haesu Lee $\mathbb{D}_{\text {, }}$ Mi Hye Kim (D), Seong Chul Jin (D), Jae Min Han (D), Jun Hyuk Park (D), \\ and Woong Mo Yang $\mathbb{D}$
} Department of Convergence Korean Medical Science, College of Korean Medicine, Graduate School, Kyung Hee University,
26 Kyungheedae-ro, Dongdaemun-gu, Seoul 02447, Republic of Korea

Correspondence should be addressed to Woong Mo Yang; wmyang@khu.ac.kr

Received 17 June 2020; Revised 8 December 2020; Accepted 22 December 2020; Published 6 January 2021

Academic Editor: Juntra Karbwang

Copyright (c) 2021 Haesu Lee et al. This is an open access article distributed under the Creative Commons Attribution License, which permits unrestricted use, distribution, and reproduction in any medium, provided the original work is properly cited.

Localized adiposity is not only a common aesthetic issue but also a health risk factor. Pharmacopuncture can be a therapeutic option for the imbalance of regional fat distribution. The tuber of Pinellia ternata has been prescribed as antitussive and expectorant as a traditional Korean medicine. This study investigated the effects of pharmacopuncture with $P$. ternata water extract (PT) on localized adiposity. Male C57BL/6J mice were fed on a high-fat diet (HFD) for 6 weeks. $100 \mu \mathrm{L}$ of $10 \mathrm{mg} / \mathrm{mL}$ of PT was injected into the left-side inguinal fat pad, while saline was injected into the right-side inguinal fat pad as self-control. Treatments were performed 3 times per week for 4 weeks. The inguinal fat weight was analyzed by dual-energy X-ray absorptiometry. PT pharmacopuncture significantly decreased the weight of the inguinal fat pad. The adipocyte size was reduced with increases of lipolytic enzymes and lipophagy-related factors by PT pharmacopuncture. There was marked inhibition of lipid accumulation content in 3T3-L1 adipocytes by PT treatment. The expressions of adipose triglyceride lipase (ATGL), hormonesensitive lipase (HSL), autophagy-related gene (ATG) 5, ATG7, and LC3 were markedly increased by PT treatments in vivo and in vitro. This study suggests that pharmacopuncture of Pinellia ternata has ameliorative effects on adiposity by lipid catabolic effects via activating both lipolysis and lipophagy in a localized region.

\section{Introduction}

Abnormal and excessive lipid storage in adipose tissues is a main characteristic of obesity, defined as a body mass index (BMI) greater than or equal to $30 \mathrm{~kg} / \mathrm{m}^{2}$ [1]. Triglyceride (TG) is a major constituent of lipid droplets (LDs) in white adipose tissues [2]. The regional distribution of TG deposition in visceral, abdominal, and subcutaneous fat, regarded as localized adiposity, is closely associated with the high risk of obesity rather than BMI [3]. For that reason, hydrolysis of TG is a vital therapeutic target not only to reduce physical inconvenience but also to improve secondary metabolic diseases such as hypertension, diabetes mellitus, cardiac disorders, and cancer in obese conditions [4].

The aesthetic treatment for obesity is commonly performed in subcutaneous adipose tissue of the abdomen [5]. Liposuction, extraction of fat tissue by suction, is one of the most frequently performed cosmetic procedures in North America [6]. While liposuction can remove the subcutaneous fat effectively with tumescent technique and small diameter cannula, complications can vary from cannula injury and infection to embolism and contour deformity and even death [7]. Phosphatidylcholine (PC) and/or deoxycholate (DC) injections have been widely used as a nonsurgical option for the correction of localized fat [8]. PC and DC injections could reduce local fat deposits by the lipolytic effects [9]. However, severe adverse effects of injection lipolysis with PC and DC have been reported such as tissue necrosis and fibrosis [10]. Pharmacopuncture, injection of natural herbs at the acupuncture point, is a new acupuncture technique in traditional Korean medicine and is able to be an alternative to liposuction and PC-DC injection [11]. Several natural herbs including Ephedra sinica Staph., Atractylodes ovata (Thunb.) DC., Panax quinquefolius L., and Eriobotrya 
japonica (Thunb.) Lindl. have been studied in pharmacopuncture for obesity [12].

The tuber of Pinellia ternata (Thunb.) Breitenb. (Araceae) has been prescribed as an antitussive and expectorant agent for throat pain, asthma, and chronic obstructive pulmonary disease in traditional Korean medicine [13]. Currently, $P$. ternata has been demonstrated to have inhibitory effects on vomiting, infection, inflammation, and cancer [14-17]. Kim et al. have reported that oral administered $P$. ternata has an antiobesity effect on Zucker rats by converting from fat depots into brown adipose tissue which is thermogenically active [18]. This study can be supported by theories of traditional Korean medicine in which $P$. ternata is known to be an efficient herb for dispelling "phlegm dampness" that is the leading cause of obesity [19]. Interestingly, a herbal formula containing $P$. ternata, Weikang Keli, has been shown to induce autophagy on the gastric cancer cell, which is confirmed by detecting biomarkers for autophagic cells including microtubule-associated protein 1 light chain 3 (LC3) [20].

However, the effect of $P$. ternata pharmacopuncture on localized obesity has not been researched. In the present study, we investigated the effect of pharmacopuncture with P. ternata water extract (PT) on inguinal fat pad of obesityinduced mice by analyzing local fat loss, adipose tissue histology, and the activities of adipose triglyceride lipase (ATGL), hormone-sensitive lipase (HSL), LC3, autophagyrelated gene (ATG) 5, and ATG7. In addition, the effects of PT on 3T3-L1 adipocyte-like cells were demonstrated by measuring the levels of lipid content, ATGL, HSL, LC3, ATG5, and ATG7 with and without the addition of lipophagy inhibitor.

\section{Materials and Methods}

2.1. Preparation of Samples. PT processed with ginger juice and alum known as Kangbanha in Korea was purchased from Dong-Yang Herb Inc. (Seoul, Korea). To avoid the toxicity of raw PT, herb manufacturer provides PT processed with ginger juice and alum in Korea. $10 \mathrm{~g}$ of PT was extracted with $150 \mathrm{~mL}$ of distilled water at $25^{\circ} \mathrm{C}$ for $2 \mathrm{~h}$. The extract was concentrated under decompression filtration. It was powdered by freeze dryer (yield: 5.76\%). The sample was stored at $-20^{\circ} \mathrm{C}$ until use.

2.2. Animal Treatment. Five-week-old male C57BL/6J mice were purchased from Raonbio Inc. (Yongin, Korea). Eight mice were housed under $22 \pm 2^{\circ} \mathrm{C}$ temperature and $50 \pm 5 \%$ humidity-controlled facility. After 1 week of housing, 10 mice were fed a high-fat diet (HFD) containing 60\% fat to induce obesity. Following 6 weeks of HFD feeding, $10 \mathrm{mg} /$ $\mathrm{mL}$ of PT was injected to left inguinal fat pad (PT), while saline was injected into the right inguinal fat pad (saline) for self-control (Figure 1(a)). The injected volume of saline and PT sample was $100 \mu \mathrm{L}$ per mice, respectively. The treatment was performed 3 times per week for 4 weeks. Body weight was measured once a week through whole animal experiments. All animal procedures were approved by the
Committee on Care and Use of Laboratory Animals of the Kyung Hee Univ. (KHUASP (SE)-18-070).

2.3. Measurement of Inguinal Fat Weight by Dual-Energy $X$-Ray Absorptiometry. At the end of the animal experiment, the mice were anesthetized. Dual-energy X-ray absorptiometry (DXA; Medikors, Seongnam, Korea) was used to analyze the fat weight of the inguinal region. The determined region was from knee to tail based on a line of the ventral spine. The fat decomposition was indicated by a red dot.

2.4. Histology. Both sides of inguinal fat pads were dissected, respectively. The specimens were fixed with $10 \%$ neutralized formalin for $24 \mathrm{~h}$. Then, fat tissues were dehydrated with gradient ethanol and xylene and embedded in paraffin. The prepared paraffin blocks were cut into $5 \mu \mathrm{m}$ using a microtome (HM355S, Thermo Scientific, UK). Each tissue slide was stained with hematoxylin and eosin to confirm the structure and diameter of the adipocyte in the inguinal fat pad. Histological changes were observed under an optical microscope (Leica DM 500; Leica, Wetzlar, Germany). Five slides of each mouse were randomly selected, and the diameter of adipocyte was measured using an automated analysis program Image (National Institutes of Health, Bethesda, MD, USA).

2.5. Measurement of Serum Toxicity. Blood samples were collected by cardiac puncture and centrifuged at $17000 \mathrm{rpm}$ for $30 \mathrm{~min}$. The supernatant was separated and used for estimation of BUN, Creatinine, AST, and ALT by enzymelinked immunosorbent assay (ELISA) according to the manufacturer's instruction.

2.6. Adipocytes Differentiation by Oil Red O Staining. 3T3-L1 murine preadipocytes were purchased from the American Type Culture Collection (Rockville, MD, USA). The cells were grown in Dulbecco's modified eagle medium (DMEM) with $10 \%$ bovine serum (BS) at $37^{\circ} \mathrm{C}$ with $5 \% \mathrm{CO}_{2}$. After cells confluence on plates (Day 0), differentiation media (MDI) containing 10\% fetal bovine serum (FBS), 1\% penicillin-streptomycin (P/S), $0.5 \mathrm{mM}$ 3-isobutyl-1-methylxanthine (IBMX), $1 \mu \mathrm{M}$ dexamethasone, and $5 \mu \mathrm{g} / \mathrm{mL}$ insulin in DMEM were incubated into cells until Day 3. The differentiation media were then changed to second differentiation media containing $10 \% \mathrm{FBS}, 1 \% \mathrm{P} / \mathrm{S}$, and $5 \mu \mathrm{g} / \mathrm{mL}$ insulin in DMEM for another 2 days. On Day 5, fresh second differentiation media were incubated again for additional 2 days. On Day 7, 3T3-L1 preadipocytes were differentiated into mature adipocytes. 1,10 , and $100 \mu \mathrm{g} / \mathrm{mL}$ of PT dissolved in distilled water were treated to MDI-induced mature adipocytes for $24 \mathrm{~h}$. In addition, $0.2 \mathrm{mM} 3$-methyladenine (3-MA), a lipophagic inhibitor, with 1,10 , and $100 \mu \mathrm{g} / \mathrm{mL}$ of PT was incubated into 3T3-L1 differentiated cells for $24 \mathrm{~h}$. Finally, the cells were fixed with 5\% neutralized formalin to measure the levels of lipid accumulation using the Oil Red O (ORO) staining method on Day 8. After incubation with $0.5 \%$ Triton X-100 for $10 \mathrm{~min}$, the cells were rinsed with $60 \%$ 


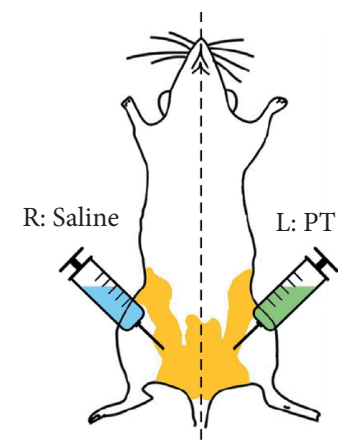

(a)

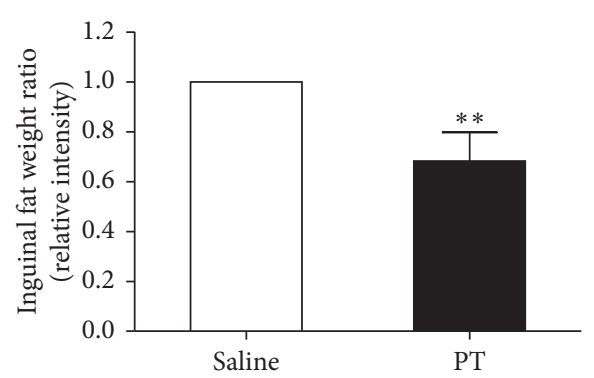

(d)

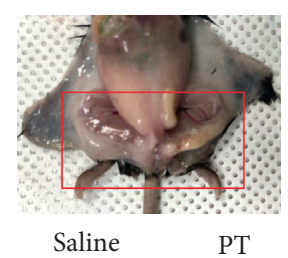

(b)

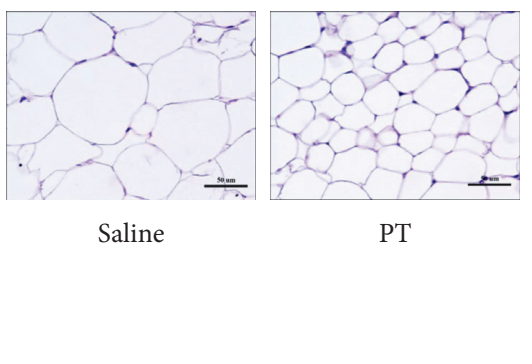

(e)

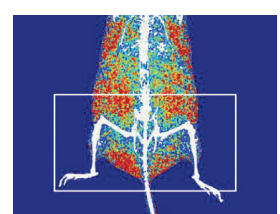

Saline $\quad$ PT

(c)

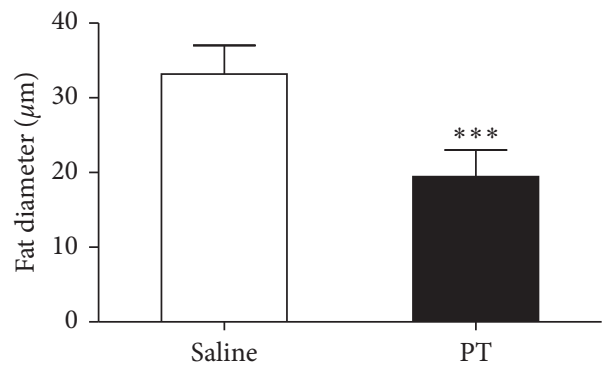

(f)

FigURE 1: Schematic diagram of sample treatment (a). Morphological changes of the inguinal fat pad (b) and representative picture from DXA (c). Red dots indicate the deposition of fat tissues visualized by the DXA program. The ratio of inguinal fat pad weight (d). The inguinal fat was weighed by DXA. Representative picture of histological changes of fat tissues (e) and quantified fat diameter of adipocyte (f). Localized adiposity and size of adipocyte were evaluated stained with H\&E. Results are presented as mean \pm standard deviation. ${ }^{* *} p<0.01$ and ${ }^{* * *} p<0.001$ vs. saline-treated right-side fat as self-control. PT, Pinellia ternata.

of isopropanol for $5 \mathrm{~min}$ and completely dried. 60\% filtered ORO working solution in distilled water was added to 3T3$\mathrm{L} 1$ cells to stain $\mathrm{LD}$ at room temperature for $2 \mathrm{~h}$. To visualize cellular LD, ORO-stained cells were observed by a microscope. In addition, relative lipid accumulation content compared to the control was quantified by an additional extraction procedure. $100 \%$ isopropanol was added to each well. The supernatant was transferred into a 96-well plate. The absorbance was measured at $500 \mathrm{~nm}$ using an ELISA microplate reader (BioTek, PA, USA).

2.7. Western Blot Analysis. Inguinal fat tissue was homogenized with tissue protein extraction buffer (Thermo Scientific, Rockford, USA) with protease inhibitor cocktails (Roche, Mannheim, Germany). The lysate was centrifuged at $17000 \mathrm{rpm}$ for $15 \mathrm{~min}$ and the supernatant was collected. The protein concentrations were calculated by Bradford assay solution (Bio-Rad, CA, USA). $10 \mu \mathrm{g}$ protein was separated on $10 \%$ SDS-PAGE and then transferred onto polyvinylidene fluoride (PVDF) membranes (Bio-Rad, CA, USA). The membranes were probed with the primary antibodies specific for $\beta$-actin, ATGL, HSL, LC3, ATG5, and ATG7 (Cell Signaling Technology, Danvers, MA, USA) at $4^{\circ} \mathrm{C}$ overnight. Secondary antibodies were incubated with horseradish peroxidase-conjugated anti-mouse or anti-rabbit (Santa Cruz Biotechnology, CA, USA) for $1 \mathrm{~h}$ at room temperature. The protein bands were detected with chem-luminescence reagents (AbClon, Seoul, Korea) by chemi-doc (Davinch-K,
Seoul, Korea). $\beta$-Actin, as a loading control, was used to normalize the levels of proteins.

2.8. Statistical Analysis. Significance was determined by oneway analysis of variance (ANOVA) and Tukey's multiple comparison tests. In all analyses, $p<0.05$ was taken to indicate statistical significance.

\section{Results}

3.1. Effects of PT Pharmacopuncture on the Inguinal Fat Weight in Obese Mice. The weight of the left inguinal fat tissues injected with PT pharmacopuncture (PT) was markedly reduced compared to that of the right inguinal fat tissues injected with saline (saline) (Figures 1(b) and 1(c)). Specifically, the fat pad administered with physiological saline was measured to be $149.2 \pm 7.5 \mathrm{mg}$, while the fat pad treated with PT weighed $107.5 \pm 16.1 \mathrm{mg}$. When the saline treatment group was converted to 1 , the relative value of PTinjected fat pad weight was estimated to $0.73 \pm 0.14$, indicating that PT pharmacopuncture decreased the weight of inguinal fat pad by about $27 \%$ compared to the saline control (Figure 1(d)).

\subsection{Effects of PT Pharmacopuncture on Histological Changes} of Inguinal Fat Tissues in Obese Mice. Hematoxylin and eosin staining was performed to confirm the histological change of localized adipose tissues and measure the diameter of 
adipocyte diameters. Injection of PT pharmacopuncture dramatically inhibited the size of adipocyte in inguinal fat pad tissues of HFD-fed obese mice (Figure 1(e)). HFD feeding induced $132.9 \pm 3.5 \mu \mathrm{m}$ of adipocyte diameter in the inguinal fat pad. The diameter of the adipocyte in adipose tissues was $79.5 \pm 7.2 \mu \mathrm{m}$ by PT injection. When the diameter of adipocyte in the saline-treated fat pad was converted to 1 , the relative value of adipocyte diameter in PT-treated fat pad was estimated to $0.6 \pm 0.06$, indicating that PT pharmacopuncture significantly decreased the adipocyte diameter of the inguinal fat pad by about $40 \%$ compared to the saline control (Figure 1(f)).

\subsection{Effects of PT Pharmacopuncture on the Expressions of} Lipolytic Enzymes in Inguinal Fat Tissues in Obese Mice. The expression of lipolysis-related factors such as ATGL and HSL was determined in fat tissues of saline and PT group, respectively. PT injection significantly increased the ATGL expression by about 3.4 times compared to saline. In addition, there was a 2.9-fold increase of HSL protein expression in the PT-injected right side of the inguinal fat pad compared to the self-control saline side (Figure 2).

3.4. Effects of PT Pharmacopuncture on Expressions of Lipophagy-Related Factors in Inguinal Fat Tissues in Obese Mice. The expression of lipophagy-related factors such as LC3, ATG5, and ATG7 was determined in fat tissues of saline and PT group, respectively. PT injection significantly increased the LC3 expression by about 2.6 times compared to salineinjected fat tissue. ATG5 and ATG7 expressions in HFDinduced fat tissues were significantly increased by treatment with PT pharmacopuncture by about 1.7 times and 1.5 times (Figure 3).

3.5. Effects of PTon Lipid Accumulation in 3T3-L1 Adipocytes. Intracellular lipid accumulation was observed by ORO staining in differentiated 3T3-L1 cells. Differentiated cells induced by MDI and additional insulin incubation showed red-stained hypertrophic adipocytes when compared to the nontreated control. There was abundance in the roundshaped intracellular LDs in differentiated 3T3-L1 cells. 1, 10, and $100 \mu \mathrm{g} / \mathrm{mL}$ of PT treatment dose-dependently inhibited the TG accumulation content by about $19.92 \%, 27.3 \%$, and $31.32 \%$ compared to differentiated adipocytes. In the presence of 3-MA, PT treatment lowered the intracellular lipid content in a dose-dependent manner by about $7.42 \%$, $20.8 \%$, and $22.02 \%$ in $1 \mu \mathrm{g} / \mathrm{mL}$ of PT $+3-\mathrm{MA}, 10 \mu \mathrm{g} / \mathrm{mL}$ of $\mathrm{PT}+3-\mathrm{MA}$, and $100 \mu \mathrm{g} / \mathrm{mL}$ of PT + 3-MA, respectively. The LD was significantly decreased even if PT extract was cotreated with 3-MA, an inhibitor of autophagy. However, the inhibition rates of lipid accumulation in cells cotreated with PT and 3-MA were lower than cells treated with only PT (Figure 4).

3.6. Effects of PT on Expressions of Lipolytic Enzymes in Differentiated 3T3-L1 Adipocytes. PT treatment in differentiated 3T3-L1 cells showed a dose-dependent effect on increases of ATGL and HSL expression. Compared with MDI-induced differentiated cells, the increased rates of ATGL by treatment with 1,10 , and $100 \mu \mathrm{g} / \mathrm{mL}$ of PT were $231 \%, 390.7 \%$, and $543 \%$. Also, cells treated with PT in the presence of 3-MA showed a similar increase \% by cells treated with only PT $(126.7 \%, 287 \%$, and $373.4 \%$ in $1 \mu \mathrm{g} / \mathrm{mL}$ of PT + 3-MA, $10 \mu \mathrm{g} / \mathrm{mL}$ of PT $+3-\mathrm{MA}$, and $100 \mu \mathrm{g} / \mathrm{mL}$ of $\mathrm{PT}+3-\mathrm{MA}$, respectively). In addition, PT 1,10 , and $100 \mu \mathrm{g} /$ $\mathrm{mL}$ treatment significantly increased the HSL expressions by about $129.7 \%, 300.5 \%$, and $352 \%$ compared to MDI-stimulated differentiated cells. There was no difference in the expression of HSL in differentiated adipocytes between only PT-treated cells and PT and 3-MA-cotreated cells (Figure 5).

3.7. Effects of PTon Expressions of Lipophagy-Related Factors in Differentiated 3T3-L1 Adipocytes. The expression of LC3 following induction of differentiation was found to be significantly increased by PT treatment in 3T3-L1 cells. In particular, treatment with $100 \mu \mathrm{g} / \mathrm{mL}$ of PT markedly raised the 1.22-fold LC3 expression. Cotreatment of PT with 3-MA also increased the expression of LC3; however, the increased rates at all concentrations of PT were less than only $100 \mu \mathrm{g} / \mathrm{mL}$ of PT-treated cells. Compared to MDI-induced differentiated cells, the expressions of ATG5 and ATG7 were significantly increased by 10 and $100 \mu \mathrm{g} / \mathrm{mL}$ of PT treatment. In cells treated with $100 \mu \mathrm{g} /$ $\mathrm{mL}$ of PT with 3-MA, a slight change of those factors was shown (Figure 6).

3.8. Effects of PT Pharmacopuncture on Body Weight in Obese Mice. Through the whole animal experiment, body weight was confirmed every 1 week to check the condition of mice. Because mice were fed with HFD, body weight was gradually increased during the induction period of obesity. Following the HFD feeding, the body weight of all mice was consistently increased during periods of the PT pharmacopuncture treatments, indicating that PT pharmacopuncture did not affect the body weight (Table 1).

3.9. Effects of PT Pharmacopuncture on Serum Hepatoxicity and Nephrotoxicity in Obese Mice. The levels of injury marker of liver and kidney were investigated to assess the potential toxic effect of PT. The serum levels of AST and ALT were $174.60 \pm 38.49 \mathrm{U} / \mathrm{L}$ and $28.40 \pm 4.72 \mathrm{U} / \mathrm{L}$ in PT-treated mice. Serum AST and ALT levels were within the normal range without hepatoxicity by PT treatment. Also, the levels of BUN and creatinine as nephrotoxic markers in PT-treated mice were $29.00 \pm 2.92 \mathrm{mg} / \mathrm{dL}$ and $0.29 \pm 0.03 \mathrm{mg} / \mathrm{dL}$, indicating that there was no toxicity such as hepatoxicity and nephrotoxicity by PT treatment to HFD-induced obese mice (Table 2).

\section{Discussion}

Localized adiposity is not only a common aesthetic issue but also a health risk factor [21]. Due to the imbalance of regional fat distribution, local administrations including 


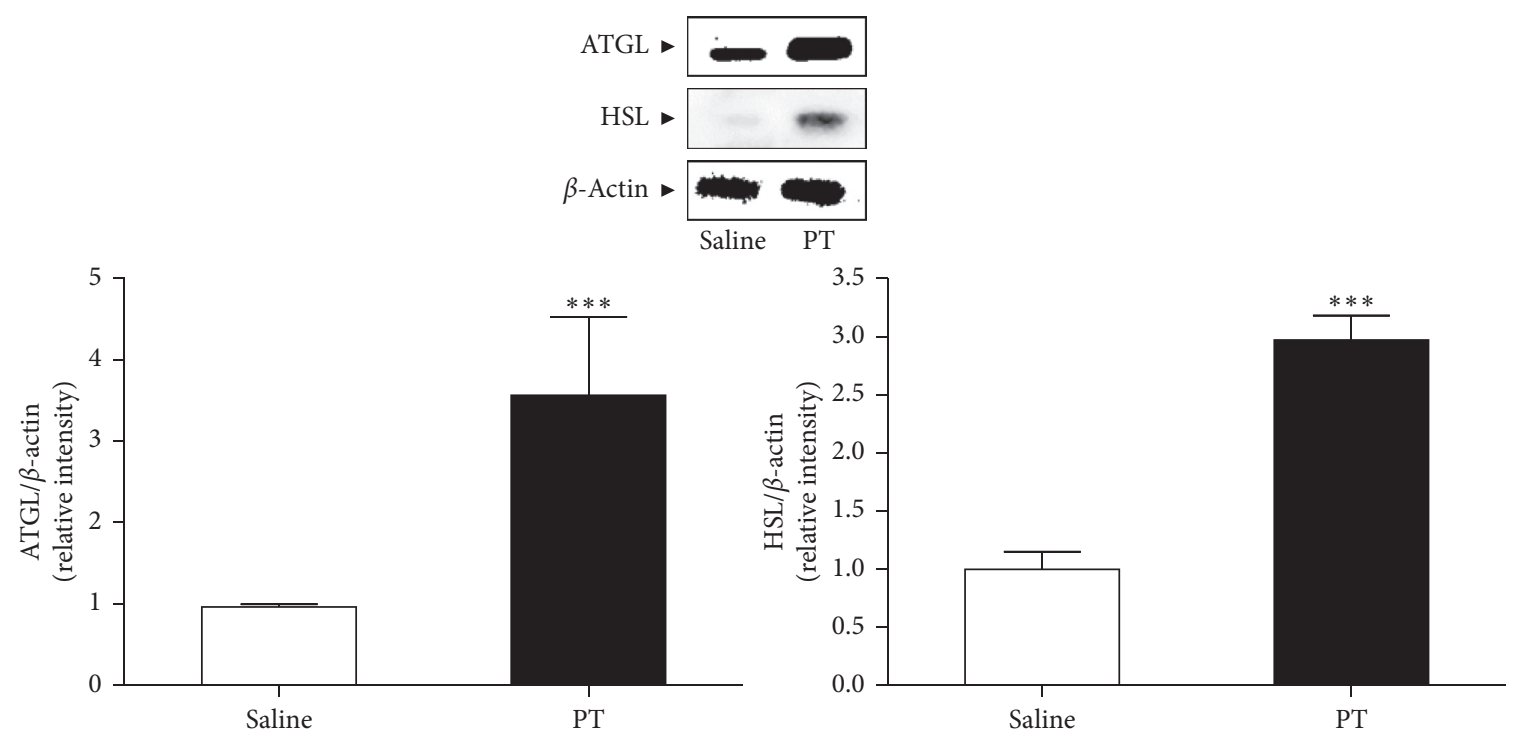

FIGURE 2: The expressions of lipolytic enzymes in inguinal fat tissues of HFD-induced obese mice. The levels of ATGL and HSL protein $(n=10)$ were visualized by Western blot analysis. Results are presented as mean \pm standard deviation. ${ }^{* * *} p<0.01$ vs. saline-treated rightside fat as self-control. The experiments were carried out in triplicate measurements. PT, Pinellia ternata; ATGL, adipose triglyceride lipase; HSL, hormone-sensitive lipase.
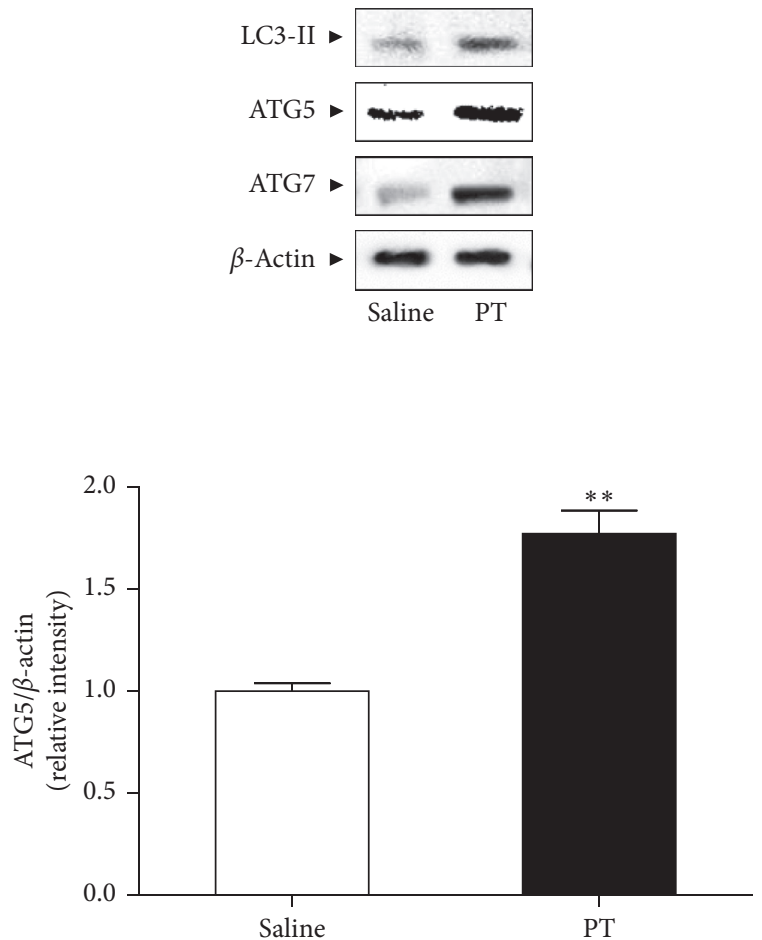
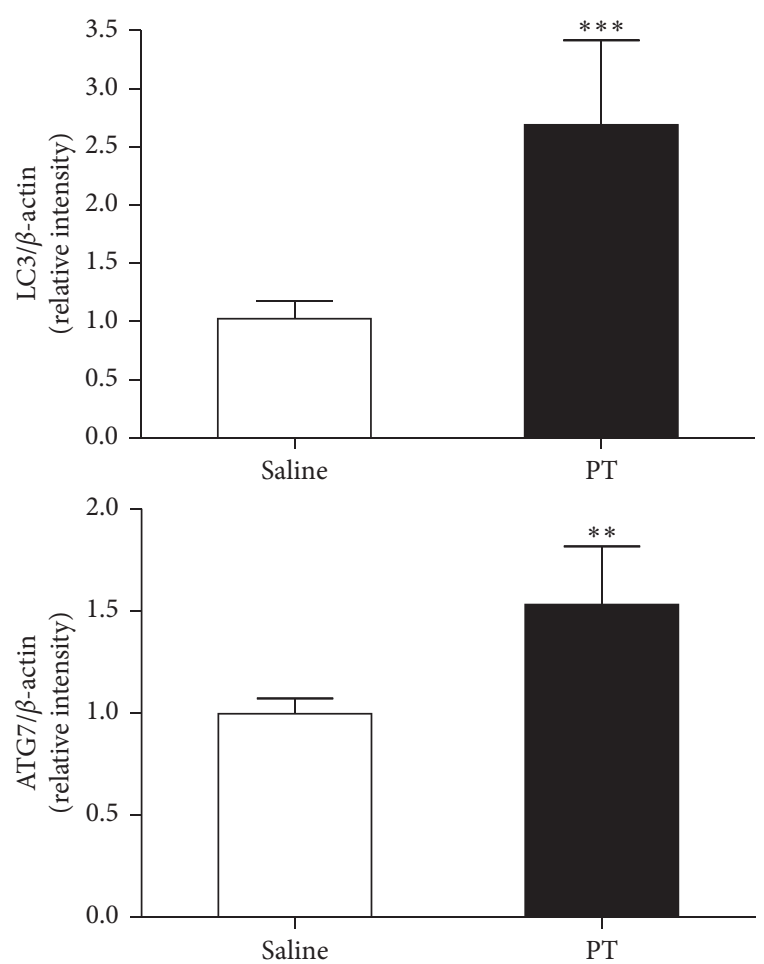

FIgURE 3: The expressions of lipophagic factors in inguinal fat tissues of HFD-induced obese mice. The levels of LC3-II, ATG5, and ATG7 protein were visualized by Western blot analysis. Results are presented as mean \pm standard deviation. ${ }^{* *} p<0.01$ and ${ }^{* * *} p<0.01$ vs. salinetreated right-side fat as self-control. The experiments were carried out in triplicate measurements. PT, Pinellia ternata; LC3-II, microtubuleassociated protein 1A/1B-light chain 3-II; ATG5, autophagy-related gene 5; ATG7, autophagy-related gene 7.

pharmacopuncture may be needed to reduce the localized expansion of fat mass [22]. Fat accumulation primarily relies on the balance between lipolysis and lipogenesis in adipose tissue [23]. Mature adipocytes are responsible for this dynamic metabolism of LDs with a unique property in cell size alteration [24]. During the fat breakdown, the size of adipocytes gets smaller as LDs shrink. However, fat synthesis is accompanied by increases in size as well as the number of 

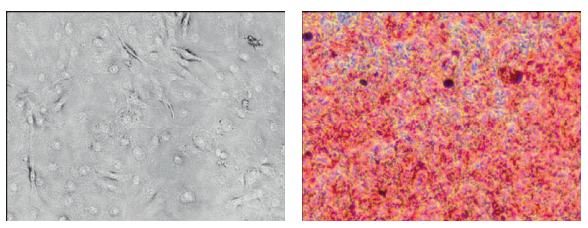

$+$

$-$

$-$

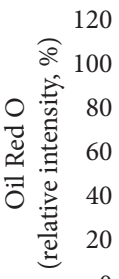

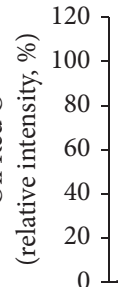

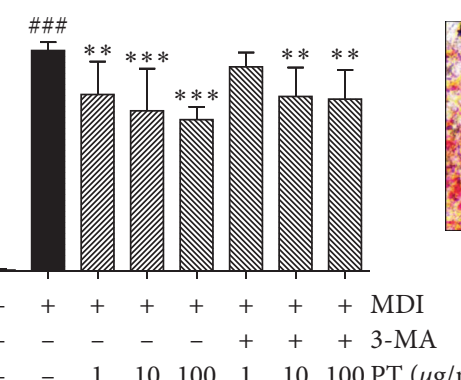

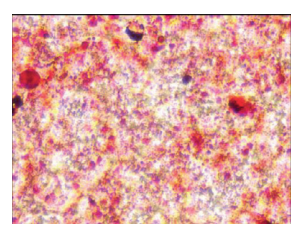

$+$

$-$

1

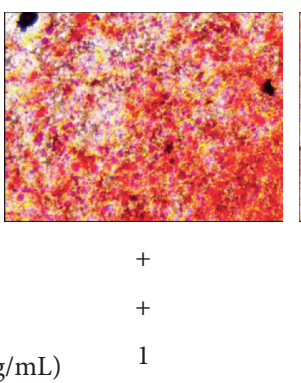

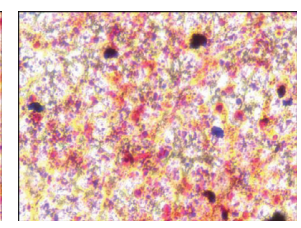

$+$

$-$

10

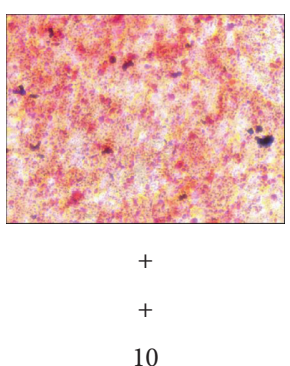

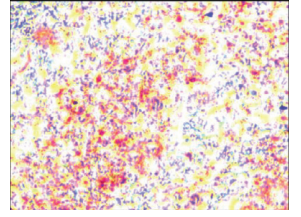

$+\quad$ MDI

$100 \quad \mathrm{PT}(\mu \mathrm{g} / \mathrm{mL})$

FIGURE 4: Intracellular lipid accumulation content in differentiated 3T3-L1 adipocytes. Intracellular lipid accumulation was conducted by ORO staining. Results are presented as mean \pm standard deviation. ${ }^{\# \# \#} p<0.001$ vs. undifferentiated cells; ${ }^{*} p<0.05,{ }^{* *} p<0.01$, and ${ }^{* * *} p<0.01$ vs. MDI-induced differentiated cells. The experiments were carried out in triplicate measurements. PT, Pinellia ternata; ORO, Oil Red O; 3-MA; 3-methyladenine.
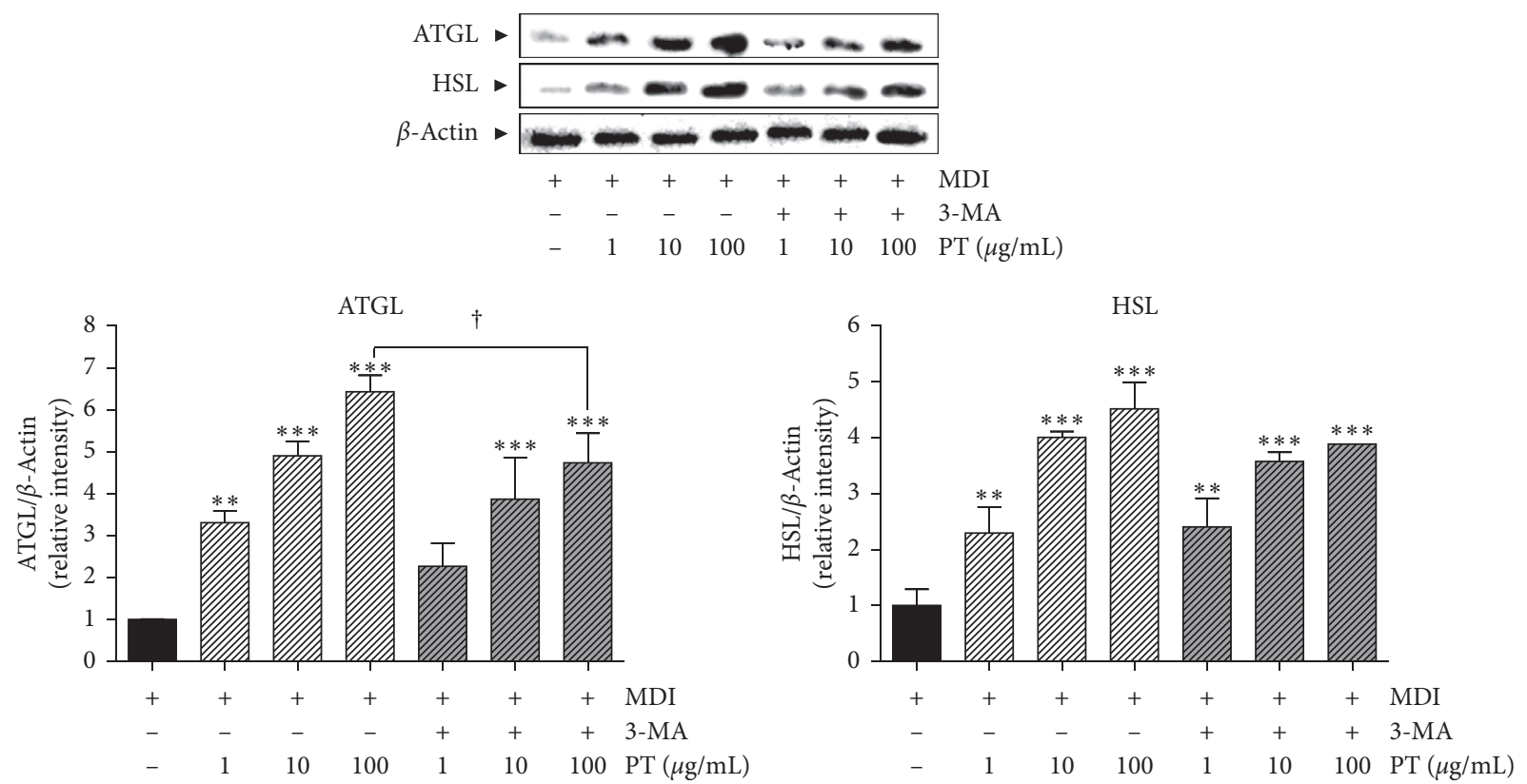

Figure 5: The expressions of lipolytic enzymes in differentiated 3T3-L1 adipocytes. The levels of ATGL and HSL protein were visualized by Western blot analysis. Results are presented as mean \pm standard deviation. ${ }^{*} p<0.05,{ }^{* *} p<0.01$, and ${ }^{* * *} p<0.01$ vs. MDI group. PT, Pinellia ternata; ATGL, adipose triglyceride lipase; HSL, hormone-sensitive lipase.

adipocytes [25]. In this study, PT pharmacopuncture was injected into the left inguinal fat pad in HFD-induced obese mice with a saline injection into the right side as self-control. PT pharmacopuncture significantly decreased the weight of inguinal fat pad and the size of adipocytes in adiposity induced mice, which means that PT pharmacopuncture seems to have effects on fat degradation.
Lipolysis is a hydrolytic process of TG in LDs to triacylglycerol-associated free fatty acids (FFAs) and glycerol for energy production in regular sequence [26]. Lipolytic activity in obesity is characterized by an overall increase with highly maintained basal lipolysis and a decrease in catecholamine-stimulated lipolysis by a defect in insulin-mediated suppression [27]. It is well established that the two 

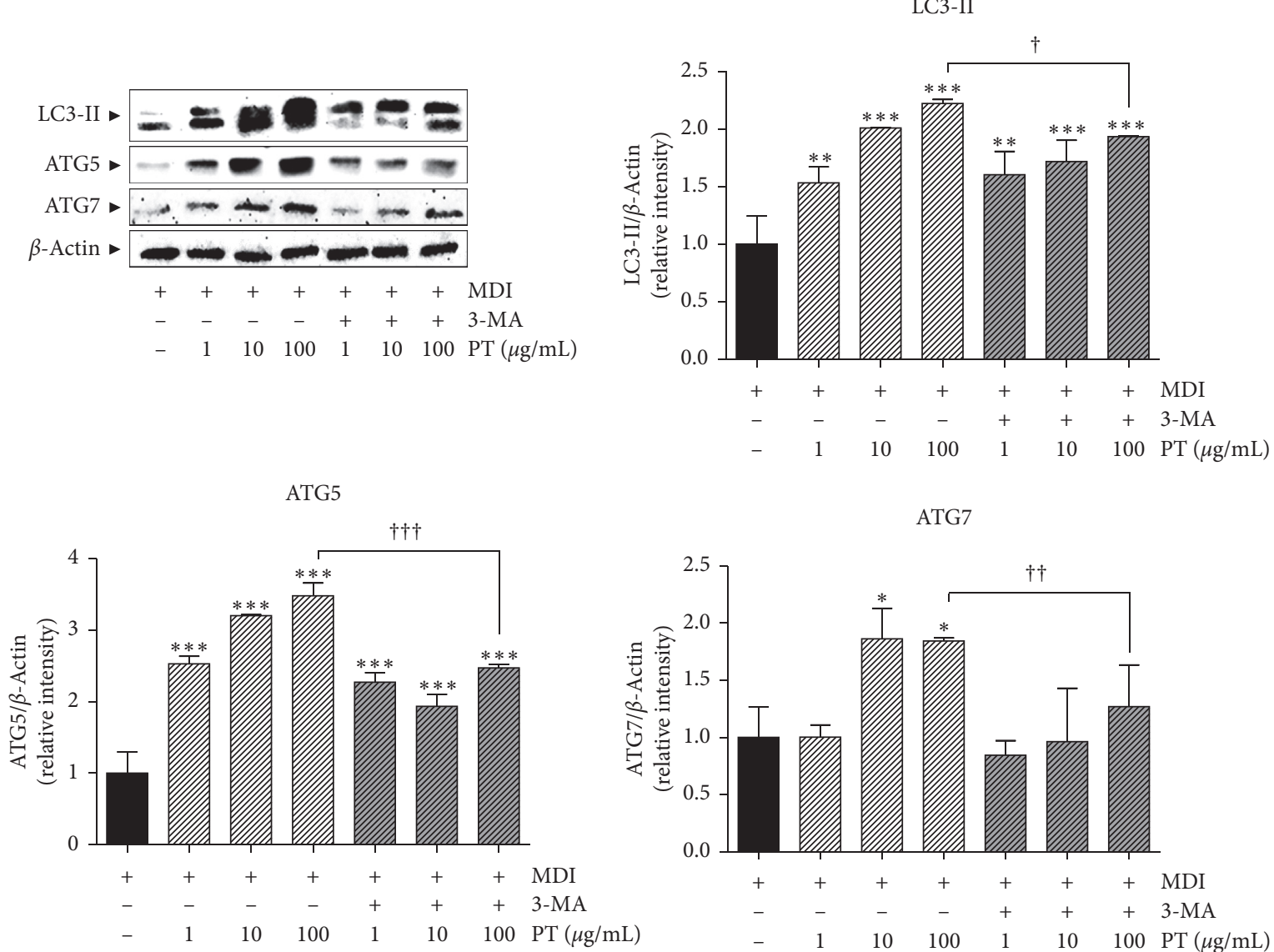

FIgURE 6: The expressions of lipophagic factors in differentiated 3T3-L1 adipocytes. The levels of LC3, ATG5, and ATG7 protein were visualized by Western blot analysis. Results are presented as mean \pm standard deviation. ${ }^{*} p<0.05,{ }^{* *} p<0.01$, and ${ }^{* * *} p<0.01$ vs. MDIinduced differentiated cells. PT, Pinellia ternata; LC3, microtubule-associated protein 1 A/1B-light chain 3; ATG5, autophagy-related gene 5; ATG7, autophagy-related gene 7.

TABLE 1: Body weight as time course in HFD-induced obese mice.

\begin{tabular}{lc}
\hline Weeks after treatment & Body weight $(\mathrm{g})$ \\
\hline-6 & $16.72 \pm 0.35$ \\
-5 & $18.63 \pm 0.42$ \\
-4 & $20.3 \pm 0.75$ \\
-3 & $21.15 \pm 0.82$ \\
-2 & $22.38 \pm 1.63$ \\
-1 & $23.78 \pm 2.08$ \\
0 & $24.27 \pm 1.49$ \\
1 & $24.35 \pm 1.86$ \\
2 & $26.07 \pm 2.50$ \\
3 & $27.20 \pm 3.04$ \\
4 & $27.08 \pm 3.29$ \\
\hline
\end{tabular}

Body weight of mice was measured every 1 week. Results are presented as mean \pm standard deviation. PT, Pinellia ternata.

major cytosolic enzymes, ATGL and HSL, mediate the hydrolysis of TG. In the present study, expressions of ATGL and HSL were markedly increased by PT pharmacopuncture indicating that PT has a lipolytic effect by local treatment.

In addition to lipolysis, lipophagy is another pathway to fat breakdown and TG utilization [28]. Since defective lipophagy is thought to have a role in the pathology of obesity with lipid breakdown, insulin sensitivity, and food
TABle 2: Serum AST, ALP, BUN, and creatinine levels in HFDinduced obese mice.

\begin{tabular}{lcc}
\hline Analyte & Value in PT-treated mice & Normal range \\
\hline AST (GOT) (U/L) & $174.60 \pm 38.49$ & $54 \sim 298 \mathrm{U} / \mathrm{L}$ \\
ALT (GPT) (U/L) & $28.40 \pm 4.72$ & $20 \sim 90 \mathrm{U} / \mathrm{L}$ \\
Creatinine (mg/dL) & $0.29 \pm 0.03$ & $<0.5 \mathrm{mg} / \mathrm{dL}$ \\
BUN (mg/dL) & $29.00 \pm 2.92$ & $16 \sim 36 \mathrm{mg} / \mathrm{dL}$ \\
\hline
\end{tabular}

Results are presented as mean \pm standard deviation. PT, Pinellia ternata. All experiments were approved by the Committee on Care and Use of Laboratory.

intake, therefore, regulating lipophagy can be a therapeutic target for adiposity and obesity [29]. In the process of lipophagy, ATGs regulate fundamental procedures of lipophagy such as autophagosome formation, transportation to the lysosome, and the fusion of the autophagosome with lysosome [30]. A pivotal component of autophagosome structure, LC3-II is formed by ubiquitin-like conjugation of LC3 and phosphatidylethanolamine, called LC3 lipidation, with the enzymic activity of ATG7 [31]. The conjugation of ATG12 with ATG5, also activated by ATG7, forms a multimeric complex with ATG16L, which contributes to the LC3 lipidation and the elongation of autophagosome membrane [32, 33]. LDs are encapsulated by an 
autophagosome and degraded by acidic enzymes of lysosomal organelle which is fused with the autophagosome subsequently [34]. The expressions of LC3-II and two essential ATG5 and ATG7 were significantly increased by PT treatment. The expressions of ATGs and LC3-II were decreased by PT in the presence of 3-MA compared to PT solo treatment in differentiated 3T3-L1 adipocytes, which is the first evidence that PT treatment may degrade TG by upregulating the lipophagy.

Taken together, PT treatment showed both lipolytic and lipophagic effects on localized adiposity in obese mice. We analyzed the degradative effects of PT on LDs with and without lipophagic activity in vitro. TG accumulation in the differentiation of 3T3-L1 adipocytes was significantly decreased by PT treatment. The expressions of lipolytic enzymes, including ATGL and HSL, and lipophagic proteins, including ATG5, ATG7, and LC3-II, were increased by PT significantly and dose-dependently in vitro, which means that PT has lipid degradative effects by activating both lipolysis and lipophagy.

\section{Conclusion}

PT pharmacopuncture showed the catabolic effects of localized adiposity by activating both lipolysis and lipophagy. These findings suggest that $P$. ternata pharmacopuncture would be an alternative treatment for reducing localized fat in obesity.

\section{Abbreviations}

3-MA: 3-Methyladenine

ATG5: Autophagy-related gene 5

ATG7: Autophagy-related gene 7

ATGL: Adipose triglyceride lipase

BMI: Body mass index

DC: Deoxycholate

HSL: Hormone-sensitive lipase

LC3: The light chain protein 3

LD: $\quad$ Lipid droplets

ORO: Oil Red O

PC: Phosphatidylcholine

PT: Pinellia ternate

TG: $\quad$ Triglyceride.

\section{Data Availability}

The data used to support the findings of this study are available from the corresponding author upon reasonable request.

\section{Conflicts of Interest}

The authors declare no conflicts of interest.

\section{Acknowledgments}

This work was supported by a National Research Foundation of Korea Grant funded by the Korean Government (NRF2019R1I1A2A01063598).

\section{References}

[1] G. H. Goossens, "The metabolic phenotype in obesity: fat mass, body fat distribution, and adipose tissue function," Obesity Facts, vol. 10, no. 3, pp. 207-215, 2017.

[2] R. Zimmermann, J. G. Strauss, G. Haemmerle et al., "Fat mobilization in adipose tissue is promoted by adipose triglyceride lipase," Science, vol. 306, no. 5700, pp. 1383-1386, 2004.

[3] I. Janssen, P. T. Katzmarzyk, and R. Ross, "Waist circumference and not body mass index explains obesity-related health risk," The American Journal of Clinical Nutrition, vol. 79, no. 3, pp. 379-384, 2004.

[4] G. A. Bray, "Medical consequences of obesity," The Journal of Clinical Endocrinology and Metabolism, vol. 89, no. 6, pp. 2583-2589, 2004.

[5] D. P. Friedmann, "A review of the aesthetic treatment of abdominal subcutaneous adipose tissue," Dermatologic Surgery, vol. 41, no. 1, pp. 18-34, 2015.

[6] M. G. Berry and D. Davies, "Liposuction: a review of principles and techniques," Journal of Plastic, Reconstructive and Aesthetic Surgery, vol. 64, no. 8, pp. 985-992, 2011.

[7] P. J. Stephan and J. M. Kenkel, "Updates and advances in liposuction," Aesthetic Surgery Journal, vol. 30, no. 1, pp. 83-97, 2010.

[8] M. E. Yagima Odo, L. C. Cuce, L. M. Odo, and A. Natrielli, "Action of sodium deoxycholate on subcutaneous human tissue," Dermatologic Surgery, vol. 33, no. 2, pp. 178-189, 2007.

[9] F. Hasengschwandtner, "Phosphatidylcholine treatment to induce lipolysis," Journal of Cosmetic Dermatology, vol. 4, no. 4, pp. 308-313, 2005.

[10] D. Duncan and R. Chubaty, "Clinical safety data and standards of practice for injection lipolysis: a retrospective study," Aesthetic Surgery Journal, vol. 26, no. 5, pp. 575-585, 2006.

[11] K.-H. Lee, Y.-Y. Cho, S. Kim, and S.-H. Sun, "History of research on pharmacopuncture in Korea," Journal of Pharmacopuncture, vol. 19, p. 101, 2016.

[12] M.-H. Nam, S.-W. Lee, H.-Y. Na et al., "Herbal acupuncture for the treatment of obesity," Journal of Acupuncture and Meridian Studies, vol. 9, no. 2, pp. 49-57, 2016.

[13] I.-S. Ok, S.-H. Kim, B.-K. Kim, J.-C. Lee, and Y.-C. Lee, "Pinellia ternata, citrus reticulata, and their combinational prescription inhibit eosinophil infiltration and airway hyperresponsiveness by suppressing CCR3," Mediators of Inflammation, vol. 2009, Article ID 413270, 10 pages, 2009.

[14] T. Maki, K. Takahashi, and S. Shibata, "An anti-emetic principle ofPinellia ternataTuber," Planta Medica, vol. 53, no. 5, pp. 410-414, 1987.

[15] X. Wang, W. Jia, A. Zhao, and X. Wang, "Anti-influenza agents from plants and traditional Chinese medicine," Phytotherapy Research, vol. 20, no. 5, pp. 335-341, 2006.

[16] L. Juan, K. Chen, B.-S. Huang, X. Lei, and X. Yu, "Anti-tumor activities of extracts from the medicinal plants pinellia ternata and pinellia pedatisecta," in Proceedings of the 2009 3rd International Conference on Bioinformatics and Biomedical Engineering, pp. 1-4, IEEE, Beijing, China, June 2009.

[17] M.-Y. Lee, I.-S. Shin, W.-Y. Jeon, H.-S. Lim, J.-H. Kim, and H. Ha, "Pinellia ternataBreitenbach attenuates ovalbumininduced allergic airway inflammation and mucus secretion in a murine model of asthma," Immunopharmacology and Immunotoxicology, vol. 35, no. 3, pp. 410-418, 2013.

[18] Y.-J. Kim, Y.-O. Shin, Y.-W. Ha, S. Lee, J.-K. Oh, and Y. S. Kim, "Anti-obesity effect of Pinellia ternata extract in Zucker rats," Biological and Pharmaceutical Bulletin, vol. 29, no. 6, pp. 1278-1281, 2006. 
[19] Y. Sui, H. L. Zhao, V. C. W. Wong et al., "A systematic review on use of Chinese medicine and acupuncture for treatment of obesity," Obesity Reviews, vol. 13, no. 5, pp. 409-430, 2012.

[20] J. Huo, F. Qin, X. Cai et al., "Chinese medicine formula "Weikang Keli" induces autophagic cell death on human gastric cancer cell line SGC-7901," Phytomedicine, vol. 20, no. 2, pp. 159-165, 2013.

[21] M. Peppa, C. Koliaki, D. I. Hadjidakis et al., "Regional fat distribution and cardiometabolic risk in healthy postmenopausal women," European Journal of Internal Medicine, vol. 24, no. 8, pp. 824-831, 2013.

[22] Y. G. Illouz, "Study of subcutaneous fat," Aesthetic Plastic Surgery, vol. 14, no. 1, pp. 165-177, 1990.

[23] S. Kersten, "Mechanisms of nutritional and hormonal regulation of lipogenesis," EMBO Reports, vol. 2, no. 4, pp. 282-286, 2001.

[24] C. Farnier, S. Krief, M. Blache et al., “Adipocyte functions are modulated by cell size change: potential involvement of an integrin/ERK signalling pathway," International Journal of Obesity, vol. 27, no. 10, p. 1178, 2003.

[25] Y. D. Tchoukalova, C. Koutsari, M. V. Karpyak, S. B. Votruba, E. Wendland, and M. D. Jensen, "Subcutaneous adipocyte size and body fat distribution," The American Journal of Clinical Nutrition, vol. 87, no. 1, pp. 56-63, 2008.

[26] M. Ahmadian, Y. Wang, and H. S. Sul, "Lipolysis in adipocytes," The International Journal of Biochemistry \& Cell Biology, vol. 42, no. 5, pp. 555-559, 2010.

[27] J. B. Albu, M. Curi, M. Shur, L. Murphy, D. E. Matthews, and F. X. Pi-Sunyer, "Systemic resistance to the antilipolytic effect of insulin in black and white women with visceral obesity," American Journal of Physiology-Endocrinology and Metabolism, vol. 277, no. 3, pp. E551-E560, 1999.

[28] R. Singh and A. M. Cuervo, "Lipophagy: connecting autophagy and lipid metabolism," International Journal of Cell Biology, vol. 2012, 2012.

[29] N. Maixner, J. Kovsan, I. Harman-Boehm, M. Blüher, N. Bashan, and A. Rudich, "Autophagy in adipose tissue," Obesity Facts, vol. 5, no. 5, pp. 710-721, 2012.

[30] Z. Yang and D. J. Klionsky, "Mammalian autophagy: core molecular machinery and signaling regulation," Current Opinion in Cell Biology, vol. 22, no. 2, pp. 124-131, 2010.

[31] T. Johansen and T. Lamark, "Selective autophagy mediated by autophagic adapter proteins," Autophagy, vol. 7, no. 3, pp. 279-296, 2011.

[32] N. Mizushima, T. Noda, T. Yoshimori et al., "A protein conjugation system essential for autophagy," Nature, vol. 395, no. 6700 , p. 395, 1998.

[33] N. Fujita, T. Itoh, H. Omori, M. Fukuda, T. Noda, and T. Yoshimori, "The Atg16L complex specifies the site of LC3 lipidation for membrane biogenesis in autophagy," Molecular Biology of the Cell, vol. 19, no. 5, pp. 2092-2100, 2008.

[34] A. R. Thiam, R. V. Farese Jr, and T. C. Walther, "The biophysics and cell biology of lipid droplets," Nature Reviews Molecular Cell Biology, vol. 14, no. 12, pp. 775-786, 2013. 